\title{
Exactitud de las tablas de composición de alimentos en la determinación de nutrientes
}

\author{
Pérez Grana R. ${ }^{1}$
}

Sanid. mil. 2013; 69 (2): 102-111; ISSN: 1887-8571

\begin{abstract}
RESUMEN
Las tablas de composición de alimentos constituyen una herramienta imprescindible para realizar una valoración nutricional de alimentos o de ingesta en las Fuerzas Armadas. Ofrecen una información semicuantitativa, más exacta para los macronutrientes o algunos elementos minerales mayoritarios (potasio, calcio y fósforo), y menos exacta para vitaminas y elementos minerales traza. Las diferencias entre las tablas de composición de alimentos y los análisis químicos son variables dependiendo de la fuente bibliográfica consultada. Así se citan unas diferencias elevadas para algunos nutrientes: fibra dietética, sodio, hierro, ácidos grasos monoinsaturados, ácidos grasos poliinsaturados y algunas vitaminas. Estas diferencias se explican por la variabilidad natural de los alimentos (alimentación animal, fertilización del suelo, grado de maduración, región de origen, etc.), tratamientos tecnológicos, plan de muestreo y otros. Su exactitud aumenta cuando se llevan a cabo estimaciones de ingesta en periodos largos de tiempo o en el caso de grandes grupos de población. Cuando se requiere una alta exactitud (estudios metabólicos, investigación clínica, etc.) el cálculo de nutrientes debería basarse en análisis químicos directos. No todas las tablas de composición de alimentos indican la variabilidad de cada nutriente (desviación estándar, límites de confianza), número de muestras analizadas, códigos de confianza para cada nutriente, que informen al usuario de su fiabilidad.
\end{abstract}

PALABRAS CLAVE: Exactitud, Tablas de composición de alimentos, Nutrientes.

\section{Accuracy of food composition tables in the determination of nutrients}

SUMMARY: The food composition tables constitute an indispensable tool to carry out a nutritional evaluation of foods or of intake in the Armed Forces. They offer semi quantitative information, more exact for the macronutrients or some major mineral elements (potassium, calcium and phosphorus), and less exact for vitamins and trace mineral elements. The differences between the food composition tables and the chemical analyses vary depending on the bibliographic sources consulted. High differences are cited for some nutrients: dietary fiber, sodium, iron, monounsaturated fatty acids, polyunsaturated fatty acids and some vitamins. These differences are explained by the natural variability of the foods (animal feeding, fertilization of the ground, maturation degree, origin region, etc.), technological treatments, sampling plan and others. Their accuracy increases when intake estimates are carried out in long periods of time or in the case of big population groups. When high accuracy is required (metabolic studies, clinical research, etc.) the calculation of nutrients should be based on direct chemical analysis. Not all food composition tables indicate the variability of each nutrient (standard deviation, confidence limits), number of analyzed samples, confidence codes for each nutrient to inform the user of their reliability.

KEY WORDS: Accuracy, Food composition tables, Nutrients.

\section{INTRODUCCIÓN}

La composición de los alimentos se considera un tema de alta prioridad por los países por el hecho existir una relación estrecha entre hábitos alimenticios, nutrición y salud de las poblaciones. Las tablas de composición de alimentos se presentan en formato impreso en papel, conocidas como tablas de composición de alimentos; o en versión electrónica, como bases de

${ }^{1}$ Tcol. Veterinario. Centro Militar de Veterinaria de la Defensa. Servicio de Bromatología y Seguridad Alimentaria. Madrid. España.

Dirección para correspondencia: Roberto Pérez Grana. Servicio de Bromatología y Seguridad Alimentaria. Centro Militar de Veterinaria de la Defensa. C/ Darío Gazapo, 3. 28024. Madrid. Tfno. 91512 2515.rpergra@oc.mde.

Recibido: 19 de junio de 2012

Aceptado: 27 de febrero de 2013 datos de composición de alimentos, con el mismo contenido en nutrientes y las mismas limitaciones. En este artículo se hace referencia, de forma general, a tablas de composición de alimentos, y a bases de datos, cuando así se exprese en la bibliografía.

Dentro del proyecto EuroFIR (http://www.eurofir.net), que incluye tablas de composición de alimentos europeas disponibles «on line», se encuentra la Base de Datos Española de Composición de Alimentos publicada por la Red BEDCA (http://bedca. net) que incluye una recopilación de datos nutricionales de diversas publicaciones y de tablas de composición de alimentos ${ }^{1,2}$.

Las tablas de composición de alimentos constituyen una herramienta de gran utilidad en las Fuerzas Armadas para realizar valoraciones nutricionales de menús o estimar la ingesta de nutrientes ${ }^{3,4}$. McCance y Widdowson's postulaban que el conocimiento de la composición química de los alimentos es el primer aspecto esencial en el tratamiento nutricional de la enfermedad y de todo estudio cuantitativo sobre la nutrición humana ${ }^{5}$. 
Tabla 1. Contenido de nutrientes en tomate y sardina comparando varias tablas de composición de alimentos.

\begin{tabular}{|c|c|c|c|c|c|c|c|c|}
\hline \multirow[b]{2}{*}{ FUENTE } & \multicolumn{4}{|c|}{ TOMATE ( Lycopersicum esculentum) } & \multicolumn{4}{|c|}{ SARDINA (Clupea pilchardus) } \\
\hline & $\begin{array}{c}\text { Fibra } \\
\mathrm{g}\end{array}$ & $\begin{array}{c}\text { Vitamina A } \\
\text { Eq. retinol } \\
\mu \mathrm{g}\end{array}$ & $\begin{array}{c}\text { Potasio } \\
\text { mg }\end{array}$ & $\begin{array}{l}\text { Niacina } \\
\text { Eq. Niacina } \\
\text { mg }\end{array}$ & $\begin{array}{l}\text { Lípidos } \\
\text { totales } \\
\mathrm{g}\end{array}$ & $\begin{array}{c}\text { Calcio } \\
\text { mg }\end{array}$ & $\begin{array}{c}\text { Fósforo } \\
\text { mg }\end{array}$ & $\begin{array}{c}\text { Vitamina A } \\
\text { Eq. Retinol } \\
\mu \mathrm{g}\end{array}$ \\
\hline 14 & $1 ’ 5$ & 300 & 280 & $0 ’ 5$ & $7 ' 5$ & 0 & 490 & - \\
\hline 11 & $0{ }^{\prime} 9$ & 94 & 270 & 0 '8 & $7 ’ 5$ & 43 & 475 & 64 \\
\hline 13 & $1 ’ 1$ & 74 & 236 & 0 '8 & $6 ’ 4$ & 74 & 270 & 39 \\
\hline 12 & $1 ’ 4$ & 94 & 250 & 1'9 & $99^{\prime} 4$ & $500^{\prime} 4$ & 258 & $62^{\prime} 9$ \\
\hline 26 & 1 '4 & 217 & - & 0 '8* & $9{ }^{\prime} 4$ & 50,4 & - & 62,9 \\
\hline 15 & 0 '95 & $\begin{array}{c}84^{\prime} 33 \\
(25-110)\end{array}$ & $\begin{array}{c}242 \\
(92-376)\end{array}$ & $\begin{array}{c}530 \\
(300-850)\end{array}$ & $\begin{array}{c}4,54 \\
\left(1^{\prime} 20-9^{\prime} 81\right)\end{array}$ & $\begin{array}{c}85 \\
(42-127)\end{array}$ & $\begin{array}{c}258 \\
(212-303)\end{array}$ & 20 \\
\hline BEDCA & - & - & - & - & - & - & - & - \\
\hline
\end{tabular}

BEDCA. Base de datos española de composición de alimentos (http://bedca.net). Consultado 5.12.2011.

* Se expresa como ácido nicotínico. (-) Dato no disponible. Las tablas de composición de alimentos alemanas proporcionan para algunos nutrientes un valor medio y valores máximo y mínimo, y la niacina se expresa en $\mu \mathrm{g}$.

Las primeras tablas de composición de alimentos aparecen en Alemania en 1878, publicadas por Josep Konig ${ }^{6}$. En 1892 se publican las tablas de composición de alimentos en USA por Atwater y Woods ${ }^{7}$. Las tablas inglesas, cuya primera edición se publica en 1940, conocidas en el mundo como «McCance y Widdowson's», han sido la mayor fuente de referencias en composición de alimentos. También la $\mathrm{FAO}^{8}$ en 1947 publica unas tablas de uso internacional.

La elaboración de las tablas de composición de alimentos requiere un proceso de compilación, en cuanto a descripción inequívoca del alimento, plan de muestreo, manejo de la muestra, método analítico, control de calidad analítico, calidad de las fuentes bibliográficas, procedimiento para estimar valores de nutrientes etc., de ahí que un factor importante en la exactitud de las tablas de composición de alimentos recae en el control de calidad del proceso compilación de datos nutricionales ${ }^{9,10}$.

Es evidente que todas las tablas de composición de alimentos no tienen la misma exactitud, llegando incluso a que se contemplen valores dispares entre algunas de amplio uso en nuestro país ${ }^{11,15}$, tal y como se puede observar en la tabla 1 .

Aunque los análisis químicos son el método preferido para producir datos de composición de alimentos y para verificar la exactitud de las tablas de composición de alimentos, en la práctica determinar cada nutriente en cada alimento tipo o en cada marca supone una gran inversión de medios y muchas veces no es posible, motivo por el cual las tablas de composición de alimentos contienen un porcentaje elevado de valores no analíticos, es decir, estimados o calculados.

\section{FACTORES QUE INFLUYEN EN LA VARIABILIDAD DE LA EXACTITUD DE LAS TABLAS DE COMPOSICIÓN DE ALIMENTOS}

\section{Nutrientes}

En las tablas de composición de alimentos se suelen encontrar diferentes tipos de valores: valores numéricos (expresados en $\mathrm{g}, \mathrm{mg}, \mu \mathrm{g}$ o ng por $100 \mathrm{~g}$ de parte comestible), valores traza,
Tabla 2. Cálculo de la energía a partir de los correspondientes factores de conversión.

\begin{tabular}{|lc|}
\hline NUTRIENTES & FACTORES DE CONVERSIÓN \\
\hline Proteína & $4 \mathrm{Kcal} / \mathrm{g}^{* *}$ \\
Grasa & $9 \mathrm{Kcal} / \mathrm{g} * *$ \\
Hidratos de carbono & $4 \mathrm{Kcal} / \mathrm{g}^{* *} ; 3,75 \mathrm{Kcal} / \mathrm{g}^{*}$ \\
Azúcares-alcohol & $2,4 \mathrm{Kcal} / \mathrm{g}$ \\
Alcohol & $7 \mathrm{Kcal} / \mathrm{g}$ \\
Ácidos orgánicos & $3 \mathrm{Kcal} / \mathrm{g}$ \\
\hline
\end{tabular}

* Valor propuesto por Southgate.

En algunos países tienen en cuenta los azúcares.

** Valores propuestos por Atwater.

valores cero y valores desconocidos. Los valores traza $(\leq 0$ 0’005 $\mathrm{mg} / 100 \mathrm{~g}$ de alimento) se pueden sustituir por cero, pero los valores desconocidos pueden ser importantes y en ningún caso se sustituirán por ceros. No obstante, algunos autores recomiendan considerar cero a efectos de cálculo ${ }^{16}$. Otros autores indican que podría dar lugar a una infraestimación de los nutrientes ${ }^{17-18}$.

En un estudio ${ }^{19}$ en el que se comparan los resultados de ingesta calculados con datos de la tabla de composición de alimentos del Departamento de Agricultura de Estados Unidos (USDA), sustituyendo los valores desconocidos con valores analíticos de la FDA, los nuevos valores de ingesta calculados, eran similares para el hierro, manganeso, zinc, potasio, magnesio, calcio, fósforo, sodio y cobre.

\section{Energía}

En cuanto al valor energético, en energía metabolizable, para una dieta variada, se utilizan los factores de conversión que se indican en la tabla 2.

Alcohol y los ácidos orgánicos (acético, cítrico, láctico, málico) para el cálculo de la energía; por ejemplo, la naranja contiene 1'2 g de ácidos orgánicos/100g de parte comestible. Fuente: Tablas de composición de alimentos Souci-Fachmann-Kraut ${ }^{15}$.

Estos valores son estimados y tienen en cuenta los diferentes calores de combustión y digestibilidades aparentes de los macronutrientes, y también las pérdidas de energía a través de la excre- 


\section{R. Pérez Grana}

ción urinaria de sustancias nitrogenadas. Southgate ${ }^{20-21}$ propone para los hidratos de carbono, 3'75 Kcal/g, que corresponde al calor de combustión de la glucosa, y se refiere, por tanto, a hidratos de carbono disponibles (expresados como monosacáridos).

Los alimentos que contienen polioles, que son alcoholes de azúcares, utilizados como edulcorantes, tienen un aporte global $2,4 \mathrm{Kcal} / \mathrm{g}$. Se asigna también un aporte energético global de ácidos orgánicos (ácido acético, cítrico, láctico y málico) de 3 $\mathrm{Kcal} / \mathrm{g}$.

En general los cálculos energéticos no se deberían tomar con una gran exactitud, ya que en los análisis de macronutrientes se calcula «proteína bruta», «grasa bruta»y, a veces, el contenido de carbohidratos se calcula por diferencia, lo que hace que se tienda a sobreestimar la energía del alimento ${ }^{22}$.

\section{Lípidos}

Debido a las recomendaciones nutricionales para reducir la ingesta de grasa, ha derivado que la grasa de las piezas de carne se elimine en la venta al por menor, lo que dificulta el trabajo de los compiladores a la hora incluir datos exactos en las tablas de composición de alimentos ${ }^{23}$. Por consiguiente, los errores al utilizar las tablas de composición de alimentos para determinar la ingesta de grasa, puede tener su origen en el tipo de carne consumida, y en la mayor o menor limpieza del tejido graso que se haga previamente a la ingesta.

Los lípidos, como analito empírico, tampoco se identifican de una manera correcta en las tablas de composición de alimentos con una especie química concreta. Así, en la valoración gravimétrica del extracto etéreo se obtienen los lípidos totales, impropiamente denominados grasa total o grasa. En esta fracción obtenida existen triacilgliceroles, o grasa, otros acilglicéridos, glucosilacilglicéridos, fosfoglicéridos, esfingolípidos, ceras, lípidos no saponificables, como son las vitaminas liposolubles A, E y K y esteroides. Esto da lugar a que la suma de los ácidos grasos no coincida con el valor de grasa que figura en las tablas. Por consiguiente, podemos hablar entonces de grasa total y grasa neta, siendo esta una forma de energía en una forma más comparable ${ }^{24}$.

\section{Agua}

El agua es el constituyente de los alimentos que más variaciones produce en el resto de los nutrientes. Las mayores variaciones se han observado en vegetales (frutas y hortalizas) dependiendo del grado de maduración y de las condiciones de su almacenamiento. Aquellos alimentos con menor porcentaje de agua producen menos variaciones en los nutrientes. Por este motivo, cuando se comparen datos nutricionales de platos preparados o de alimentos es importante asegurar que las comparaciones se realizan con el mismo contenido en agua.

\section{Proteínas}

Los valores de proteínas de muchos alimentos varían en un corto intervalo, y por tanto, las tablas de composición de alimentos predicen la ingesta proteica con una exactitud razonable (error máximo del $7 \%)^{25}$. Las proteínas se expresan en las tablas de composición de alimentos como proteína total, ya que en los análisis por el método Kjeldahl se obtiene nitrógeno que se multiplica por un factor correspondiente dependiendo del alimento, por ejemplo 5'87 para cereales, 6'58 para leche, etc., o por un factor genérico igual a 6’25, en el análisis de platos preparados o a efectos de etiquetado nutricional. Por tanto, no se considera el nitrógeno no proteico, lo que constituye una fuente de error, y además todas las proteínas no contienen por término medio un $16 \%$ de nitrógeno. La determinación del nitrógeno total incluye sustancias nitrogenadas no proteicas (urea, creatinina, ácido úrico, bases púricas y pirimídicas, etc.), lo que puede conducir a error en los alimentos ricos (pescados, frutas, hongos, verduras y hortalizas). De esta manera cuando se comparan datos de composición de alimentos de diferentes fuentes, los usuarios deberían considerar el factor de conversión del nitrógeno y si el nitrógeno no proteico ha sido excluido del contenido de proteína. En este sentido las tablas del Ministerio de Sanidad y Consumo, con un contenido de 68 alimentos, discriminan entre nitrógeno total y nitrógeno proteico ${ }^{26}$.

\section{Fibra dietética}

La fibra dietética incluye ciertos polisacáridos (celulosa, hemicelulosa, pectinas, oligosacáridos, gomas, mucílagos, almidón resistente) y compuestos fenólicos polimerizados (lignina), y se desglosa en fibra soluble e insoluble con distintas repercusiones fisiológicas.

La fibra dietética es un buen ejemplo de un componente en el cual el método analítico puede afectar al resultado. Así, los valores obtenidos usando el método de la AOAC enzimáticogravimétrico ${ }^{27}$, oficial en numerosos países, que mide también lignina, almidón resistente y otros carbohidratos no digestibles, son más altos que aquellos métodos que miden polisacáridos no amiláceos $^{28}$. No obstante, el método de la AOAC, a pesar de su fiabilidad, presenta fuentes de error cuando se aplica alimentos líquidos o bebidas ${ }^{29}$.

\section{Hidratos de carbono}

Los hidratos de carbono (HC) en las tablas de composición de alimentos pueden estar expresados y estimados por diferentes métodos. El porcentaje de hidratos de carbono disponibles, se puede calcular por diferencia, según la expresión:

$\% \mathrm{HC}=100 \square(\%$ agua $+\%$ proteína $+\%$ grasa $+\%$ fibra dietética $+\%$ cenizas $+\%$ etanol)

Esta expresión puede incluir también el \% de ácidos orgánicos disponibles (ácido cítrico, ácido láctico, ácido málico) ${ }^{30-31}$. Los métodos por «diferencia» pueden incorporar algún error derivado de la determinación de cada componente. Esta medida tiende a ser reemplazada por la medida directa de los azúcares y almidones y fibra dietética ${ }^{32}$, hecho que debe ser tenido en cuenta al usar las tablas de composición de alimentos.

\section{Minerales}

Como regla general los nutrientes mayoritarios $(\mathrm{Ca}, \mathrm{K}, \mathrm{Mg}$, P) varían de forma estrecha en los alimentos sin procesar, aunque en el caso del fósforo resulta difícil su estimación exacta de- 


\section{Exactitud de las tablas de composición de alimentos en la determinación de nutrientes}

bido a la cantidad de fósforo que aportan los aditivos añadidos, especialmente en los derivados de carne de ave ${ }^{33}$. En cambio, los constituyentes traza ( $\mathrm{Fe}, \mathrm{Cu}, \mathrm{Zn}, \mathrm{Mn}, \mathrm{I}, \mathrm{Cr}$, etc.) tienden a ser más variables y menos precisos. Por ejemplo, el contenido de zinc de vegetales, leche y frutas puede estar influenciado por las condiciones ambientales. Así, en áreas urbanas existe un incremento significativo de las concentraciones, más de 2-3 veces, en comparación con niveles normales. También los granos de cereales producidos en áreas urbanas del Este de Alemania muestran un contenido de zinc, entre un 12-15\% (3790 $\mu \mathrm{g} / 100)$, más alto que en zonas agrarias no contaminadas $(3370 \mu \mathrm{g} / 100 \mathrm{~g})$, y aquellos productos cultivados alrededor de industrias del metal mostraron un incremento del 40-60\%(5.430 $\mu \mathrm{g} / 100 \mathrm{~g})^{34}$.

En alimentos procesados se hace difícil, por ejemplo, predecir los valores de sodio exactamente, ya que su contenido está sujeto a amplias variaciones y el error en los cálculos dietéticos basado en tablas de composición de alimentos puede alcanzar $\pm 50 \%$.

Cuando se usen tablas de composición de alimentos de otros países $^{36}$ pueden existir posibles errores si se toma su valor y se aplica a tablas de un país donde los alimentos no están fortificados, y dentro de un país, los niveles de nutrientes varían con la marca y con la formulación de los productos ${ }^{37}$.

\section{Vitaminas}

En las tablas de composición de alimentos se observan valores muy dispares, lo que puede estar relacionado, aparte de la propia variabilidad natural, con la labilidad al calor, pérdidas durante el almacenado, cocinado y con los distintos métodos analíticos utilizados (microbiológicos o químicos), o con las distintas fuentes bibliográficas utilizadas para obtener datos. Por ejemplo, los valores de folato utilizando ensayos microbiológicos con Streptococcus faecalis son más bajos que aquellos obtenidos con Lactobacillus rhamnosus (caseii) ${ }^{38}$.

Tampoco se puede asumir que se obtendrán resultados comparables en la determinación de vitaminas del grupo B por ensayos microbiológicos y HPLC. Por consiguiente, se espera que las tablas de composición de alimentos contengan un valor aproximado. En el caso de la vitamina $\mathrm{C}$ y ácido fólico, que se caracterizan por una gran variabilidad natural y ser muy lábiles al calor, las tablas de composición de alimentos dan una orientación semicuantitativa. Otro ejemplo es la vitamina A, que se almacena en tejidos tales como el hígado y la alimentación ocasiona amplias variaciones en el contenido.

\section{OTROS FACTORES DE VARIABILIDAD QUE INFLUYEN EN LA EXACTITUD DE LAS TABLAS DE COMPOSICIÓN DE ALIMENTOS}

Un factor clave en la exactitud de las tablas de composición de alimentos es el muestreo, seleccionando apropiadas muestras. El plan de muestreo debe incluir principios de demografía del país, tipo de alimento, origen, marca, cuota de mercado, equilibrio entre áreas urbanas y rurales, etc. Se ha comprobado que las muestras tomadas en almacenes al por menor o en mercados al por mayor o en lugares de producción, resultan representativas de la distribución nacional. Por ejemplo, si se trata de hallar la composición química de vitamina $\mathrm{C}$ de una fruta, se tomaran muestras de varias variedades de distintas zonas geográficas en distintas épocas del año y durante varios años. El análisis de un número adecuado de muestras (individuales o compuestas) permite un cálculo más exacto de la media del contenido en nutrientes.

Un modelo de muestreo consiste en combinar muestras refrigeradas y congeladas de diferentes marcas y de peso estándar para obtener una muestra compuesta ${ }^{39}$. Y el número de muestras necesarias para construir una muestra compuesta se puede determinar a través de la ecuación estadística de Cochran y Holden ${ }^{40}$

$$
\mathrm{n} \geq \frac{t^{2}}{r^{2}} \mathrm{CV} 2
$$

Donde, t es igual a 1,96 para un nivel de significación de 0,05; $\mathrm{n}$, es el tamaño de la muestra; $r$, nivel de precisión del análisis químico; $\mathrm{CV}$, coeficiente de variación basado en la variabilidad del nutriente en el alimento. Así, por ejemplo, para un nutriente en el que desea obtener un $10 \%$ de precisión en el análisis químico y con una variabilidad esperada, que en el caso del $\mathrm{P}, \mathrm{K}$, Mg sería del $15 \%$, el tamaño de la muestra sería de 9 muestras. La variabilidad y el nivel de precisión determinan el número de muestras, que podría ser elevado. En un estudio realizado en hortalizas, la variabilidad para la vitamina $\mathrm{K}$, expresada en $\%$ $\mathrm{CV}$, alcanza en la zanahoria el $17^{\prime} 6 \%$ y en la col 6’3 $\%{ }^{41}$.

A partir de un protocolo de muestreo en carne de pollo realizado en Italia para la elaboración de tablas de composición de alimentos ${ }^{42}$, se toman entre 16 y 32 muestras considerando la variable peso de la canal y edad, lo que indica que el número de muestras depende de diversas variables. Según el USDA al menos 25 muestras serían necesarias para mejorar la fiabilidad de la estimación del valor medio de un nutriente ${ }^{43}$. En la tabla 3 se muestra el número de muestras, en tres bases de datos europeas, para obtener resultados de beta-caroteno en vegetales.

Existen diversos factores derivados de los alimentos y del proceso de compilación que pueden afectar a la exactitud de las tablas de composición de alimentos (Tabla 4).

Dependiendo de la raza de vacuno y de la alimentación, varía la composición de grasa y ácidos grasos y el contenido de grasa intramuscular ${ }^{44}$, pudiendo incluso, no ser la misma que hace años debido a la influencia de factores genéticos.

También se demuestra que según el tipo de alimentación los lípidos tienen una correlación negativa con la humedad y proteí$\mathrm{na}^{45}$. Otro ejemplo muy significativo es la grasa de peces, dependiendo que procedan de su hábitat natural o criado en acuicultura con piensos industriales.

Existen muchos ejemplos en la naturaleza en los que las variaciones climáticas y estacionales pueden determinar modificaciones en la composición de alimentos de origen animal y vegetal ${ }^{46-49}$; por ejemplo, el contenido de grasa de la sardina (Clupea pilchardus) y arenque (Clupea harengus membras) varía con al estación ${ }^{50}$. A partir de los análisis de sardinas pescadas en la costa portuguesa, se alcanza un mínimo en marzo (1'2 g de grasa/100g) y un máximo en septiembre $(18,4 \mathrm{~g} \text { de grasa/100g })^{51}$. El nivel más alto de ácidos grasos poliinsaturados y EPA (ácido eicosapentaenoico, C20:5, n-3) ocurren en septiembre cuando el pescado tiene su máximo contenido de grasa, y los más altos 


\section{R. Pérez Grana}

Tabla 3. Contenido de $\beta$-caroteno $(\mu \mathrm{g} / 100 \mathrm{~g}$ ) en vegetales por $100 \mathrm{~g}$ de parte comestible. http://www.eurofir.net.

\begin{tabular}{|c|c|c|c|}
\hline Alimento & Dinamarca & Francia & Noruega \\
\hline Brécol, congelado & $610(270-950)^{*}$ & - & \\
\hline Brassica oleraca var. Italica & $\mathrm{N}^{\circ}$ de muestras: 1 & & 360 \\
\hline Brécol, crudo & $533(69,0-1450)$ & - & \\
\hline Brassica oleraca var. Italica & $\mathrm{N}^{\circ}$ de muestras: 9 & & 360 \\
\hline Zanahoria, cruda & $11.000(5650-16300)$ & $7000(2700-9650)$ & \\
\hline Daucus carota $L$. & $\mathrm{N}^{\mathrm{o}}$ de muestras: 9 & $\mathrm{~N}^{\circ}$ de muestras: 43 . A & 7800 \\
\hline Zanahoria, congelada & $12.770 * *$ & $9040(5300-12770)$ & \\
\hline Daucus carota $L$. & $\mathrm{N}^{0}$ de muestras: - & $\mathrm{N}^{\circ}$ de muestras: $2 . \mathrm{C}$ & 8600 \\
\hline Espinaca, cruda & $4186(1610-5750)$ & 4600 (3500-5626). C & \\
\hline Spinacea oleracea $L$. & $\mathrm{N}^{o}$ de muestras: 21 & $\mathrm{~N}^{\circ}$ de muestras: 3 & - \\
\hline Coliflor, cruda & $40(18,0-102)$ & $24(8-40)$ & \\
\hline Brassica oleracea var. botrytis & $\mathrm{N}^{\circ}$ de muestras: 8 & $\mathrm{~N}^{\circ}$ de muestras: 2. C & 8 \\
\hline Coliflor, congelada & $18 * *$. & $12(7-18)$ & \\
\hline Brassica oleracea var. botrytis & $\mathrm{N}^{\circ}$ de muestras: - & $\mathrm{N}^{\circ}$ de muestras: $2 . \mathrm{C}$ & 50 \\
\hline $\begin{array}{l}\text { Guisante verde, congelado } \\
\text { Pisum sativum L. }\end{array}$ & $\begin{array}{c}357(350-364) \\
\mathrm{N}^{\circ} \text { de muestras: } 4\end{array}$ & - & 737 \\
\hline
\end{tabular}

* Dato obtenido de USDA National Nutrient Database for Standard Reference, Release, 20. (-) Dato no disponible. La base de datos nutricional noruega no indica el número de muestras ni los valores máximos y mínimos.

** Dato obtenido por cálculo a partir de diferentes valores analíticos. A, C: Códigos de confianza. A (Valor más fiable).

Tabla 4. Factores de variabilidad que afectan a la exactitud de las tablas de composición de alimentos.

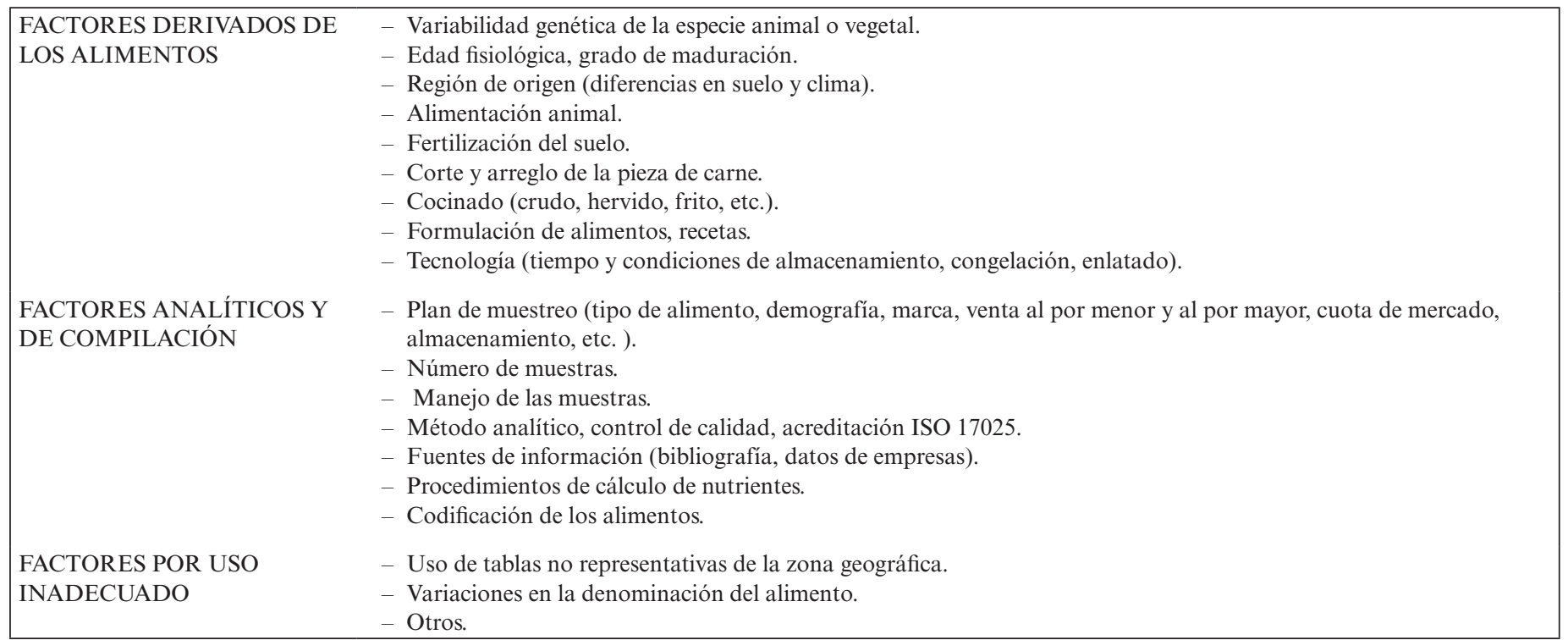

niveles de DHA (ácido docosahexaenoico, C22:6, n-3) fueron en agosto.

En el caso de vegetales, por ejemplo, el contenido de folato de las fresas depende significativamente de los efectos del cultivo, madurez y año de cosecha ${ }^{52}$.

La composición de nutrientes de un alimento también se ve afectada por el almacenamiento, procesado, cocinado, etc., es decir, por tecnología industrial y doméstica. El USDA ${ }^{53}$ ha publicado los factores de retención para vitaminas y minerales en diversos alimentos y para varios métodos de cocinado, usando datos de muestras crudas y cocinadas según la fórmula:
Factor de retención $=$ Contenido de nutrientes por $\mathrm{g}$ de alimento cocinado $\mathrm{x}$ gramos de alimento cocinado/Contenido en nutrientes por $\mathrm{g}$ de alimento crudo $\mathrm{x}$ gramos de alimento crudo.

Los diferentes procesos culinarios afectan de forma distinta al contenido de nutrientes, especialmente, el perfil de ácidos grasos como consecuencia de la fritura ${ }^{54}$, el perfil de aminoácidos e incluso en el contenido total de proteínas.

Durante la cocción de la carne hay pérdidas de tiamina (73$100 \%$ ), así como pérdidas de calcio, sodio, potasio, magnesio y fósforo, mientras que el hierro y zinc aumentan ${ }^{55}$ pero en general la cocción de alimentos apenas produce pérdidas de minerales ${ }^{56}$. 


\section{Exactitud de las tablas de composición de alimentos en la determinación de nutrientes}

La fritura se ve afectada por el tipo de aceite e introduce modificaciones en la composición de los lípidos, que en los pescados provoca incluso diferencias entre especies de pescado ${ }^{57}$. Este tratamiento afecta a la grasa total y a los ácidos grasos; por ejemplo, las sardinas crudas y la caballa presentan un importante contenido de AGP (ácidos grasos poliinsaturados, n-3 (24 y $16^{\prime} 6 \mathrm{~g} / 100 \mathrm{~g}$ de grasa, respectivamente), pero decrece significativamente durante la fritura $\left(6{ }^{\prime} 6\right.$ y 5'4 g/100g de grasa, respectivamente). En cambio en el salmón a pesar de tener una más baja cantidad de ácido eicosapentaenoico y ácido docosahexaenoico en muestras crudas, era la mejor fuente de estos ácidos grasos después de la fritura ( $1^{\prime} 7 \mathrm{~g} / 100 \mathrm{~g}$ de alimento). También el contenido de colesterol se afecta de forma diferente por la fritura, incremento o descenso, dependiendo de la especie de pescado.

Asimismo, se observan variaciones en los lípidos durante el mantenimiento en caliente ${ }^{58}$. Una medida ideal para evitar sesgos, consiste en documentar la retención de nutrientes pesando los alimentos antes y después del cocinado ${ }^{59}$.

En otro estudio similar ${ }^{60}$, los ácidos grasos monoinsaturados de la sardina se incrementan 4,2 veces cuando se fríe en aceite de girasol y 10 veces en aceite de oliva. Lo anterior explica porque resulta difícil realizar estimaciones de los nutrientes de los alimentos sometidos a tratamientos culinarios ${ }^{61}$.

Otro factor que afecta a la exactitud de las tablas de composición de alimentos, es un uso inadecuado de las mismas por el usuario. Los errores se relacionan con la identificación equivocada de los alimentos, con variaciones en la denominación en alimentos aparentemente similares que se usan en otra lengua, con un tratamiento inadecuado de los valores desconocidos, etc., en cuyo caso se puede infraestimar o sobreestimar un nutriente crítico. Otros errores son debidos al uso de tablas que no sean representativas de una determinada zona geográfica, uso de tablas de composición de alimentos poco actualizadas, ya que deberían reflejar los cambios del mercado alimentario ${ }^{62}$.

Se han hallado diferencias significativas $(p<0,0001)$ en la estimación de las ingestas medias de macronutrientes, vitamina $\mathrm{C}$ y potasio en 1.314 individuos y 5 grupos de alimentos (patatas, frutas, cereales, hortalizas y legumbres) utilizando 3 bases de datos de composición de alimentos (inglesa, francesa y danesa), existiendo una diferencia máxima de 33'6 mg entre las bases de datos para la vitamina C; 5'6 g para la fibra, dependiendo del método de análisis utilizado; $28^{\prime} 4 \mathrm{~g}$ para los carbohidratos y 673 kcal para la energía ${ }^{63}$. Tales diferencias se atribuyen a las diferencias en la composición de alimentos entre países.

\section{ANÁLISIS QUÍMICO VERSUS TABLAS DE COMPOSICIÓN DE LOS ALIMENTOS}

En general la calidad de los datos de las tablas de composición de alimentos depende de número de muestras analizadas y si éstas son representativas de todos los alimentos con la misma denominación, del método analítico usado y de la exactitud de los datos ${ }^{64}$.

No se han encontrado diferencias significativas realizando un análisis de la varianza para calorías, proteína y grasa, entre los análisis químicos y los datos de dos bases nutricionales, sobre 4 menús de dietas de investigación ricas en grasa y bajas en gra- $\mathrm{sa}^{65}$. Para carbohidratos totales, se halla una diferencia significativa $(\mathrm{p}<0,0001)$ entre los datos del laboratorio y las dos bases de datos nutricionales. La fibra dietética presentaba diferencias significativas $(\mathrm{p}<0,00028)$ para un test $\mathrm{t}$ de valores pareados, siendo los valores de los análisis químicos del $176 \%$ y $172 \%$ respecto a los valores calculados para dietas bajas y ricas en grasa.

Otros autores ${ }^{66}$ realizan una estimación en 36 menús para detener la hipertensión, sobre 13 nutrientes (energía, grasa total, grasa saturada, grasa monoinsaturada, grasa poliinsaturada, carbohidratos, colesterol, proteína, calcio, potasio, magnesio, hierro y sodio) utilizando 4 tablas de composición de alimentos. Las tablas de composición de alimentos variaban significativamente en las desviaciones medias en relación a los valores de análisis químicos para ácidos grasos saturados, monoinsaturados y poliinsaturados, potasio, magnesio y hierro $(\mathrm{p}<0,5)$; sin embargo, las diferencias eran menores del $10 \%$. Las desviaciones absolutas, que estiman el efecto combinado de sesgo y precisión, fueron significativamente diferentes, entre tablas de composición de alimentos, para energía, ácidos grasos saturados y poliinsaturados. Las diferencias significativas entre tablas de composición de alimentos y análisis químicos variaban un $15 \%$, excepto para el hierro. Por último los autores señalan que las 4 tablas de composición de alimentos eran comparables en exactitud y precisión.

En cambio a partir de análisis químicos por duplicado de grasa total y ácidos grasos, se concluye que dos tablas de composición de alimentos no estiman por igual la ingesta de nutrientes de 39 participantes ${ }^{67}$.

En otro trabajo ${ }^{68}$ sobre la alimentación de los cadetes de la Academia de la Armada Italiana, se realiza la validación de las tablas de composición de alimentos con análisis químicos directos para estimación de macronutrientes (energía, carbohidratos, lípidos, proteína y fibra dietética). Se toman muestras por duplicado durante los 5 primeros días de la semana y durante 2 semanas. Las muestras se congelan a $-20{ }^{\circ} \mathrm{C}$ hasta el momento del análisis. Cada análisis se realiza por triplicado y se obtiene un valor medio. Las proteínas se determinaron por el método Kjeldahl, los hidratos de carbono por el método Fehling y método UV enzimático, la grasa, mediante hidrólisis previa y extracción con éter y la fibra total por el método enzimático gravimétrico (AOAC, 1990). Para la energía el sesgo absoluto era $\leq 077 \%$. Las tablas de composición de alimentos infraestiman ligeramente los carbohidratos (sesgo $\leq 4$ '1\%), lípidos ligeramente sobreestimados ( $\operatorname{sesgo} \leq 2^{\prime} 9 \%$ ), las proteínas sobreestimadas (sesgo $\leq 62 \%$ ). Los autores del estudio concluyen que la estimación de la fibra dietética con tablas de composición de alimentos no es válida al obtenerse un sesgo $\leq 31^{\prime} 6 \%$.

Los valores calculados del estudio anterior en cuanto a energía, proteína, grasa total y carbohidratos, también concuerdan con los valores obtenidos por análisis químico en otras dietas militares ${ }^{69}$. En las tablas 5 y 6 se presentan las diferencias en la composición de macronutrientes entre análisis químicos y resultados de tablas de composición de alimentos en una receta de platos preparados y en raciones individuales de campaña.

Cuando se comparan valores calculados frente a valores analizados para comprobar la fiabilidad del procedimiento de cálculo de recetas para 8 minerales, 6 vitaminas y colesterol en 6 platos mixtos, se hallan diferencias menores del $10 \%$, excepto para el cobre que era más alto que los valores analizados. Las 
Tabla 5. Comparación de datos obtenidos experimentalmente con datos de tablas de composición de alimentos.

\begin{tabular}{|c|c|c|c|c|c|c|}
\hline \multirow{2}{*}{ MACRONUTRIENTES } & \multicolumn{5}{|c|}{ AN y Referencia Bibliográfica } & \multirow{2}{*}{ DF } \\
\hline & 58 & 11 & 12 & 13 & 14 & \\
\hline Humedad & 74,1 & 74,8 & 77,6 & 78,1 & - & -4 \\
\hline Proteína & 5,9 & 5,2 & 5.8 & 5,2 & 4,6 & 1,3 \\
\hline Grasa total & 3,4 & 1,9 & 2,0 & 1,95 & 2,1 & 1,5 \\
\hline Carbohidratos & 10,5 & 10,7 & 11,6 & 15,4 & 12,5 & $-4,9$ \\
\hline Fibra dietética & 6,4 & 6,7 & 4,9 & 4,1 & 4,4 & 2,3 \\
\hline Kcal/100g & 96,2 & 81,1 & 87,6 & 99,5 & 88,1 & 15,1 \\
\hline $\mathrm{KJ} / 100 \mathrm{~g}$ & 402,1 & 339,3 & 366,5 & 416,30 & 368,6 & 62,8 \\
\hline
\end{tabular}

Receta: guisantes congelados $(82,5 \%)$, patata (13,8\%), jamón serrano $(1,4 \%)$, aceite de oliva (orujo) $(1,4 \%)$, sal $(0,5 \%)$.

AN: Análisis nutricional. DF: Diferencia máxima entre valores analizados y calculados.

Tabla 6. Comparación de datos obtenidos experimentalmente (valores medios) de raciones individuales de combate con datos de tablas de composición de alimentos

\begin{tabular}{|c|c|c|c|c|c|c|c|c|}
\hline MUESTRAS & MACRONUTRIENTES & $\mathrm{AN}^{1}$ & TCA 1 & TCA 2 & TCA 3 & TCA 4 & BEDCA & $\mathrm{DF}$ \\
\hline \multirow{3}{*}{$\begin{array}{l}\text { Melocotón en almíbar } \\
\qquad(\mathrm{n}=6)\end{array}$} & Humedad & 86,4 & 76,6 & 79 & 82,55 & - & - & 9,8 \\
\hline & Proteína & 0,5 & 0,4 & 0,4 & 0,38 & 0,4 & - & 0,1 \\
\hline & Grasa total & 0,2 & $\operatorname{Tr}$ & 0,1 & 0 & 0 & - & 0,2 \\
\hline \multirow{3}{*}{$\begin{array}{l}\text { Atún blanco en aceite } \\
\qquad(\mathrm{n}=7)\end{array}$} & Humedad & 54,0 & 51,5 & 62 & 59,5 & - & - & $-8,0$ \\
\hline & Proteína & 19,7 & 24 & 23,8 & 26,2 & 25 & - & $-6,5$ \\
\hline & Grasa total & 23,1 & 24,5 & 12,6 & 10,15 & 20 & - & 12,9 \\
\hline \multirow{3}{*}{$\begin{array}{l}\text { Sardinas en aceite } \\
\qquad(\mathrm{n}=5)\end{array}$} & Humedad & 49,5 & 62,8 & 60 & 55,67 & - & 62.82 & $-13,3$ \\
\hline & Proteína & 19,6 & 22,2 & 22,6 & 24,1 & 21 & 23,99 & $-4,5$ \\
\hline & Grasa total & 26,3 & 15 & 15,75 & 18,7 & 12 & 13,9 & 14,3 \\
\hline \multirow{3}{*}{$\begin{array}{l}\text { Cóctel de frutas en } \\
\text { almíbar } \\
(\mathrm{n}=7)\end{array}$} & Humedad & 85,0 & 83,8 & 82 & 82,2 & - & - & 3 \\
\hline & Proteína & 0,3 & 0,4 & 0,4 & 0,4 & - & - & $-0,1$ \\
\hline & Grasa total & 0,2 & $\operatorname{Tr}$ & 0,07 & 0,1 & - & - & 0,1 \\
\hline
\end{tabular}

${ }^{1}$ Fuente: Centro Militar de Veterinaria de la Defensa. Madrid. España.

AN: Análisis nutricional. n: Número de muestras analizadas. TCA: Tablas de composición de alimentos. DF: Diferencia máxima entre valores analizados y calculados. TCA 1: Moreiras y col., ${ }^{11}$ 2007. TCA2: Mataix Verdú y col., ${ }^{12} 2009$. TCA 3: Farran y col., ${ }^{13}$ 2004. TCA 4: Jiménez Cruz y col., ${ }^{14} 1997$. BEDCA: Base de datos española de composición de alimentos (http://bedca.net). Fecha de consulta: 5.12.2011. (-) Dato no disponible.

vitaminas mostraban diferencias menores del $20 \%$, con excepción de la vitamina $\mathrm{B} 12^{70}$. Otros estudios han comparado valores estimados con valores analizados para fibra dietética y ácido linoleico (C18:2, n-6) en 31 productos alimenticios, llegando a la conclusión que los métodos de cálculo sobreestimaban ambos nutrientes alrededor de un $10 \%{ }^{71}$.

También en estudios de ingestas de adolescentes ${ }^{72}$ se constatan diferencias significativas entre valores calculados y análisis de fibra soluble, insoluble y fibra total utilizando un método enzimático-gravimétrico, obteniéndose una diferencia media del $20{ }^{\prime} 5 \%(\mathrm{p}<0,05)$.

En un trabajo finlandés ${ }^{73}$ sobre dietas de reducción de peso y cuyo objetivo era comparar los valores calculados, con una base datos nutricionales, con los valores de 15 muestras analizadas, ésta proporcionaba una razonable buena estimación de la ingesta de proteína, grasa, ácidos grasos, fibra dietética, calcio, magnesio, potasio y manganeso; una moderada o incierta estimación para la ingesta de hierro, sodio, zinc y selenio; y pobre estimación de la ingesta de cobre, molibdeno, cadmio y plomo.

Los hallazgos anteriores se relacionan con los hallados por otros autores en China, Corea, Japón, Malasia y Filipinas ${ }^{74,75}$. Se concluye que la estimación de la ingesta de elementos minerales, en mujeres no fumadoras, con tablas de composición de alimen- tos presenta, en el caso de hierro, importantes desviaciones respecto a los análisis químicos, y en general no es posible, excepto para el sodio, realizar una estimación precisa de las ingestas de potasio, magnesio, cobre, zinc, calcio y fósforo.

En otro estudio ${ }^{76}$ se comparan los parámetros de platos cocinados de una residencia de ancianos (grasa total, ácidos grasos, colesterol, alfa-tocoferol y minerales) utilizando tablas de composición de alimentos (Mataix y col., 1995; MaCance y Widdowson's, 1991) con análisis químicos (31 platos cocinados y 12 ingredientes). Los valores calculados dan una buena estimación para energía y grasa, lo que coincide con los datos de otros autores en menús de restaurantes ${ }^{77}$. También se obtiene una buena estimación de ácidos grasos poliinsaturados, colesterol, potasio y fósforo. En contraste los valores obtenidos se tiende a infraestimar ácidos grasos saturados, ácidos grasos monoinsaturados, calcio, magnesio y sodio, y sobreestimar alfa-tocoferol y hierro. Las diferencias más grandes entre valores calculados y analizados se encuentran para ácidos grasos monoinsaturados, ácidos grasos saturados y alfa-tocoferol (38, 48 y 49\%, respectivamente). Entre los minerales determinados por plasma acoplado por inducción, las diferencias más grandes fueron observadas para el hierro y sodio (36 y $56 \%$, respectivamente). Para las ingestas de magnesio las diferencias entre los valores medios cal- 


\section{Exactitud de las tablas de composición de alimentos en la determinación de nutrientes}

culados y los valores analizados dan un rango del $11 \%$. En otro estudio emprendido en una población anciana ${ }^{78}$, se concluye que las tablas de composición de alimentos no son apropiadas para estimar la ingesta de calcio, magnesio y fósforo.

En un estudio realizado por una empresa de catering en platos preparados 79 se analizaron ácidos grasos, vitamina E, tiamina y riboflavina. Los valores calculados se obtienen a partir de receta con tablas de composición de alimentos (dos italianas, una inglesa y una de USA). Cuando se usaba más de una para el cálculo, no había diferencias significativas entre las tablas de composición de alimentos ni entre los valores analíticos y las tablas de composición de alimentos, y se concluye que las tablas de composición de alimentos son bastante adecuadas para la evaluación nutricional en grupos de población, aunque se permita una variación estacional de los ingredientes.

En un estudio piloto sobre 60 comidas y recetas para esco$\operatorname{lares}^{80}$, se valoró si los nutrientes calculados utilizando una base de datos reflejaba adecuadamente los resultados de los análisis químicos de grasa total, grasa saturada, grasa monoinsaturada, grasa poliinsaturada y sodio. La media de los valores calculados era similar a los valores analizados para grasa total (10'9 $\pm 7^{\prime} 6$ y $\left.11^{\prime} 5 \pm 7^{\prime} 4 \mathrm{~g} / 100 \mathrm{~g}\right)$, grasa saturada (4'24 $\pm 3{ }^{\prime} 25$ y 4'53 $\pm 3,26$

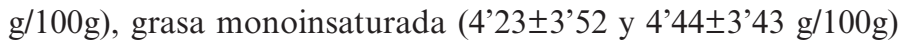
y ácidos grasos poliinsaturados (1'57 \pm 1 '79 y 1'47 \pm 1 ' $89 \mathrm{~g} / 100 \mathrm{~g}$ ). El valor global de sodio calculado en relación al analizado era significativamente más alto $(468 \pm 286$ y $307 \pm 182 \mathrm{mg} / 100 \mathrm{~g})$.

En un estudio iniciado en Japón para validar tablas de composición de alimentos para estimar la ingesta de minerales ${ }^{81}$, se toman muestras por duplicado y se halla un riesgo de sobreestimación del $26 \%$ para el magnesio, del $22 \%$ para el hierro y del $22 \%$ para el manganeso, y la estimación debe ser cuidadosa para el calcio, hierro y sodio.

En otro trabajo ${ }^{82}$ sobre el análisis de ácidos grasos en 3 dietas finesas (dieta grasa saturada, dieta grasa monoinsaturada y dieta grasa poliinsaturada) se obtienen los siguientes porcentajes en relación a los valores calculados: ácidos grasos (85-113\%), ácidos grasos monoinsaturados (65-89\%) y ácidos grasos poliinsaturados (83-99\%). Los valores de beta-caroteno y retinol en las dietas monoinsaturadas y poliinsaturadas eran del 50-60 $\%$ de los valores calculados.

Finalmente, otros autores señalan que las tablas de composición de alimentos tienen una buena exactitud cuando se realizan estimaciones de ingestas con grandes poblaciones o con pocos individuos durante un periodo de tiempo prolongado ${ }^{83}$.

\section{CONCLUSIONES}

Las diferencias en los valores de nutrientes entre las tablas de composición de alimentos, y entre éstas y análisis químicos, se explican por diferencias debidas a la variabilidad natural de los alimentos (especialmente en alimentos con alto contenido en agua), cambio en la composición de los alimentos durante la preparación, diferentes métodos analíticos usados, posibles inexactitudes del método analítico cuando los nutrientes se presentan en pequeña concentración, cálculo nutricional a partir de alimentos crudos, etc. Por todo ello las tablas de composición de alimentos ofrecen una información semicuantitativa, más exacta para los macronutrientes o algunos constituyentes inorgánicos (potasio, calcio y fósforo). Aunque los datos difieren entre las publicaciones, las principales diferencias afectan a la fibra dietética, sodio, ácidos grasos, vitaminas y elementos minerales traza. La exactitud mejora cuando se estiman ingestas en grandes poblaciones o en pocos individuos en largos períodos de tiempo.

Para evitar una sobreestimación o infraestimación de nutrientes, los valores desconocidos se deberían estimar mediante cálculo o completando con datos bibliográficos.

Conforme avanza la metodología analítica y con el desarrollo de nuevos materiales certificados de referencia para medir la exactitud en alimentos, es posible que las tablas de composición de alimentos sean más exactas.

Cuando se requiere una alta exactitud, como en estudios metabólicos, investigación clínica, etc., los datos nutricionales deberían basarse en análisis químicos directos cuando las ingestas se relacionan con medidas fisiológicas.

No todas las tablas de composición de alimentos incluyen un índice de medida de la variabilidad de cada nutriente (SD, límites de confianza, incertidumbre), número de muestras analizadas o un código de confianza de cada nutriente.

\section{BIBLIOGRAFÍA}

1. Martínez-Burgos MA, Martínez-Victoria I, Milá R, Farrán A, Farré R, Ros G, Yago MD et al.. Building a unified Spanish food database according to EuroFIR specifications. Food Chem 2009; 113:784-788.

2. Ros G, Martínez de Victoria E, Farrán A. Spanish food composition database: A challenge for a consensus. Food Chemistry 2009; 113:789-794.

3. Klicka MV, King N, Lavin P, Askew EW. Assessment of dietary intakes of cadets at the US Military Academy at West Point. J Am Coll Nutr 1996; 15 (3): 273-282.

4. Pérez Grana R. Perfil nutritivo de los menús de invierno de una empresa de restauración colectiva. Medicina Militar 2004; 60(4):230-236.

5. Holden JM, Harnly JM, Beecher CR. Composición de los alimentos. Conocimientos actuales sobre nutrición. En. Bowmann BA, Russell RM. Washington: ILSI, 2003:645-657.

6. Castanheira I, Robb P, Owen L, Der Boer H, Schmit J, Ent H, Calhan MA. A proposal to demonstrate a harmonized quality approach to analytical data production by EuroFIR. J Food Comp Anal 2007; 20:725-732.

7. Haytowitz DB, Lemar LE, Pehrsson PR. USDA's nutrient databank system a diverse sources. J Food Comp Anal 2009; 22:433-441.

8. Polacchi W, Weissell R, Marrow S, Mazar E. Derivation of nutrient values for FAO statistical databases. J Food Comp Anal 2002; 15:515-522.

9. Westernbrink S, Oseredczk M, Castanheira I, Roe M. Food composition databases: The EuroFIR approach to develop tools to assure the quality of the data compilation process. Food Chem 2009; 113 (3):759-767.

10. Hartmann BM, Vásquez-Caicedo AL, Bell S, Krems C, Brombach C. The German nutrient database: Basis for analysis of the nutritional status of the German population. J Food Comp Anal 2008; 21:S115-S118.

11. Moreiras O, Carbajal A, Cabrera L, Cuadrado C. Tablas de composición de alimentos. Madrid: Pirámide. $11^{\text {a }}$ Edición. 2004.

12. Mataix Verdú, J. Tablas de composición de alimentos. Instituto de nutrición y tecnología de los alimentos. Granada: Editorial Universidad de Granada. $5^{a}$ Edición. 2009.

13. Farran A, Zamora R, Cervera P. Tablas de composición de alimentos del CESNID. Madrid: McGraw-Hill Interamericana. Ediciones Universidad de Barcelona, 2004.

14. Jiménez Cruz A, Cervera Ral P, Bacardí Gascón M. Tablas de composición de alimentos. Barcelona: Sandoz Nutrición, 1997.

15. Souci Fachmann Kraut. Die Zusammenesetzung der Lebensmitted Nährwest- Tabellen.5.,Revidierte und ergänzte Auflage. Sttugart. Germany: Medipharm Scientific Publishers, 1994. 


\section{R. Pérez Grana}

16. Ortega Anta RM, López Sabaler AM, Requejo Marcos AM, Andrés Carbajales P. La composición de los alimentos. Madrid: Editorial Complutense, 2004:1-95.

17. Southgate DAT. Trace elements: databases and food composition compilations. Food Chem 1992; 43:289-293.

18. Briony T. Manual of dietetic practice. Oxford: Blackwell Publishing Company. $3^{\circ}$ Edition, 2001: 25-28.

19. Pennington JA, Wilson DB. Daily intake of nine nutritional elements: Analyzed vs calculated values. J Am Diet Assoc, 1990; 90: 375-381.

20. Southgate DAT, Durning JVGA. Calorie conversion factors. An experimental reassessment of the factors used in the calculation of the energy value of human diets. Br J Nutr 1970; 24:517-535.

21. Southgate DAT. The relationship between food composition and available energy. Joint FAO/WHO/UNU Expert Consultation on energy and protein requirements. 1981.

22. Koivistoinen PE, Asp NG, Englyst HN, Hudson G, Hyvönen L, Kaltio H et al. Memorandum on terms, definitions, and analytical procedures of protein fat and carbohydrates in food for basic composition data: issues and recommendations. Food Chem 1996; 57:33-35.

23. Cobiac L, Droulez V, Leppard P, Lewis J. Use external fat with to describe beef and lambs cuts in food composition tables. J Food Comp Anal 2003; 16:133-145.

24. Hyvönen L. Approach to fat analysis of foods. Food Chemistry 1996; 57:23-26.

25. Mañas Almendros M, Martínez de Victoria Muñoz E, Yago Torregrosa MD. Tablas de composición de alimentos y bases de datos nutricionales. En: Gil A. Tratado de Nutrición. Tomo II. Composición y calidad nutritiva de los alimentos. $2{ }^{\text {a }}$ Edición. Madrid: Editorial Panamericana, 2010:613-638.

26. Requejo Marcos AM, Ortega Anta RM. Tablas de composición de alimentos españoles. Madrid: Ministerio de Sanidad y Consumo, 1999.

27. Garbelotti ML, Marsiglia DAP, Torres EAFS. Determination and validation of dietary fiber in food by the enzymatic gravimetric method. Food Chem 2003:83:469-473.

28. Galleher DD, Schneeman BO. Fibra alimentaria. En: Ziegler EE, Filer LJ. Conocimientos actuales sobre nutrición. $7^{\mathrm{a}}$ Edición. Washington: ILSI Press, 1997: 95-105.

29. Saura-Calixto FD, Goñi Cambodón I, Martin Albarrán C, Pulido Ferrer R Fibra dietética en la cerveza: contenido, composición y evaluación nutricional. Madrid: Centro de Información Cerveza y Salud, 2002:15-24.

30. Kirchhoff E. Online-publication of the German food composition table «Souci-Fachmann-Kraut» on the internet. J Food Comp Anal 2002; 15:465472 .

31. Souci-Fachmann-Kraut. Tablas de composición de alimentos. Zaragoza: Editorial Acribia, 1991.

32. Uusitalo U, Kronberg-Kippila C, Aronsson CA, Schakel S, Schoen S, Mattison I et al. Food Composition database harmonization for between-country comparisons of nutrient data in the TEDDY Study. J Food Comp Anal 2011; 24:494-505.

33. Sullivan CM, Leon JB, Sehgal AR. Phosphorus-Containing food additives and the accuracy of nutrient renal patients. J Ren Nutr 2007; 17: 350-354.

34. Scherz H, Kirchhoff E. Trace elements in foods: Zinc contents of raw foodsA comparison of data originating from different geographical regions of the world. J Food Comp Anal 2006; 19:420-433.

35. Rojas Hidalgo E. Dietética. Principios y aplicaciones. Madrid: Aula Médica, 1998:89-95.

36. Patterson KY, Philips KM, Horst RL, Byrdwell WC, Exler J, Lemar LE et al. Vitamin D content and variability in fluid milks from a US Department of Agriculture nationwide sampling to update values in the National Nutrient Database to Standard Reference. J Dairy Sci 2010; 93:5082-5090.

37. Holden JM, Harnly JM, Beecher GR. Composición de los alimentos. En: Bowmann BA, Russell RM. Conocimientos actuales sobre nutrición. Washington: ILSI. 8 ${ }^{\text {a }}$ Edición, 2003:645-657.

38. Southgate DAT. Food composition data. Compilation, uses and limitations En: Sadder MJ, Strain JJ, Caballero B. Encyclopedia of human nutrition. London: Academic Press. Vol. 2, 1999:850-857.

39. Church SM. EuroFIR synthesis report No 7: Food composition explained. British Nutrition Foundation Nutrition Bulletin 2009; 34:250-272.

40. Parpinel M, Gnagnarella P, Salvini S. Proposal for the validation of the Italian food composition database. J Food Comp Anal 2000; 13:511-523.
41. Piironen V, Koivu T. Quality of vitamin K analysis and food composition data in Finland. Food Chem 2000; 68: 223-226.

42. Marletta I, Lucarini M, Ruggeri S, Carnovale E. Food Composition Database in Italy: Problems and perspectives. J Food Comp Anal 2000; 13:611-618.

43. Holden JM, Bhagwat SA, Patterson KY. Development of a Multi-nutrient Data Quality Evaluation System. J Food Comp Anal 2002; 15:339-348.

44. Cuvelier C, Clinguart A, Hocquette JF, Cabaraux JF, Dufrasne I, Istasse L et al. Comparison of composition and quality traits of meat from young finishing bulls from Belgium glue, Limousin and Aberdeein augus breeds. Meat Sci 2006; 74(3):522-531

45. Roue A, Macedo FAF, Visen Fainer JV, Souzan NE. Muscle composition and fatty and profile in lambs fattened in drylot or pasture. Meat Sci 1999; 51(4):279-399

46. Hulshol PJM, van Roekel-Jansen T, van de Bovenkamp P, West EC. Variation in retinol and carotenoid content of milk and milk products in The Netherlands. J Food Comp Anal 2006; 19:61-67.

47. Hardisson A, Rubio C, Baez A, Martín M, Alvarez R, Díaz E. Mineral composition of the banana (Musa acuminata) from the island of Tenerife. Food Chem 2001; 73:153-161.

48. Bandarra NM, Batista I, Nunes ML, Empio JM, Christie WW. Seasonal changes in liquid composition of sardine (Sardina pilchardus). J Food Sci 1997; 62:40-42.

49. Tahir M, Vandenberg A, Chibbar RN. Influence of environment on seed carbohydrates in selected lentil cultivars. J Food Comp Anal 2011; 24:596-602.

50. Aro T, Tahvonen R, Mattila T, Nurmi J, Sivonen T, Kaltio H. Effects of season and processing on oil content and fatty acids of Baltic herring (Clupea harengus membras). J Agric Food Chem 2000; 48(12):6085-6093.

51. Farran Codina A, Boatella Riera J. Tablas y bases de datos de composición de los alimentos. En: Hernández Rodríguez M, Sastre Gallego A. Tratado de nutrición. Madrid: Díaz de Santos, 1999:345-361.

52. Stralsjo LM, Witthoft CM, Sjöholm MI, Jägerstad M. Folate content in strawberries (Fragariaxanansa): Effects of cultivar, ripeness, year of harvest, storage, and commercial processing. J Agric Food Chem 2003; 7:128-133.

53. Nutrient Data Laboratory, Beltsville Human Nutrition Research Center, (BHNRC), Agricultural Research Service (ARS), Department of Agriculture (USDA). Table of nutrient retention factors. Release 6. 2007. http://www.ars. usda.gov/nutrient data. Fecha de consulta: 29.11.11.

54. Rosario Ramírez M, Estévez M, Morucuerda D, Cava R. Effect of the type of frying culinary fat on volatile compounds isolated in fried pork loin chops by using SPME-GC-MS. J Agric Food Chem 2004; 52(25):7637-7643.

55. Gerber N, Scheeder MRL, Weuk C. The influence of cooking and fat trimming on the actual nutrient intake from meat. Meat Sci 2009; 81 (1):148-154

56. Mataix Verdú J, Astiasarán Anchorena I. Tecnología alimentaria. En: Mataix Verdú J. Tratado de nutrición y alimentación. Volumen 2. Barcelona: Océano, 2009:517-534.

57. Bhori AM, Harzallah HJ, Dhibi M, Bouthel I, Hammani M, Chaouch A Nutritional fatty acid quality of raw and cooked farmed and wild sea bream (Sparus aurata). J Agric Food Chem 2010:507-512.

58. Bello Gutiérrez J. Tablas de composición para platos cocinados. Madrid: Díaz de Santos. 1998.

59. Murphy EW, Griner PE, Gray BC. Comparisons of methods for calculating retention of nutrients in cooked foods. J Agric Food Chem 1975; 23(6):1153-1157.

60. Sánchez- Muñiz FJ, Viejo JM, Medina R. Deep-frying of sardines in different culinary fats. Changes in the fatty acid composition of sardines and frying fats. J Agric Food Chem 1992; 40:2252-2256.

61. Gall KL, Otwell WS, Koburgier JA, Appledorf H. Effects of four cooking methods on the proximate, mineral and fatty acid composition of fish fillets. Food Sci 1983; 48(4):1068-1074.

62. Ovasksinen ML, Valsta LM, Lauronen J. The compilation of food analysis values as a database for dietary studies-the Finish experience. Food Chem 1996; 57:133-136.

63. Charrondiere UR, Vignat J, Moller A, Ireland J, Beckers W, Church S et al. The European Nutrient database (ENDB) for nutritional epidemiology. J Food Comp Anal 2002; 15:435-451.

64. Pennington JAT. Applications of food composition data: Data sources and considerations for use. J Food Comp Anal 2008; 21: S3-S12.

65. Simonatis N, Simpson MT, Hatcher BS, Hagan DW. Comparison of the nutrient composition of research diets using computer and laboratory nutrient analysis. J Am Diet Assoc 1997: 97(9): Supplement A107. 


\section{Exactitud de las tablas de composición de alimentos en la determinación de nutrientes}

66. McCullough ML, Karanja NM, Pao-Hwa L, Obarzanek E, Phiplips MK, Laws RL et al. Comparison of 4 nutrient databases with chemical composition data from the dietary approaches to stop hypertension trial. J Am Diet Assoc 1999; 99 (8):S45-S53.

67. Primorac Lj, Mandic ML, Klapec T, Folivarski K, Perl A, Sudar R. Adequacy of food composition database to estimate fat and fatty acid intake. International J Food Sci Nutr 2000; 51:25-32.

68. Bedogni G, Bernini C, Gatti G, Severi S. Comparison of food composition tables and direct chemical analysis for the assessment of macronutrient intake in military community. Int J Food Sci Nutr 1999; 50 (1):73-79.

69. Pograjc L, Stibilj V, Scancar J, Jamnik M. Determination of macronutrients and some essential elements in the slovene military diet. Food Chem 2010; 122: $1235-1240$.

70. Schakel SF, Buzzard MI, Gebhardt S. Procedures for estimating nutrient values for food composition database. J Food Comp Anal 1997; 10:102-114.

71. Westrich B, Buzzard IM, Gatewood KC; McGovern PG. Accuracy and efficiency of estimating nutrient values in commercial food products using mathematical optimization. J Food Comp and Anal 1994; 7:223-239.

72. Djukic N, Sobajic S, Dordevic B, Miletic I, Gajic I. Dietary fiber intake of adolescents living in a boarding school in north-eastern part of Serbia: comparison of analyzed and calculated values. Int J Food Sci Nutr 2009; 60(6):14 22.

73. Hakala P, Marniemi J, Knuts LR, Kumpulainen J, Tahvonen R, Piaani S Calculated vs analysed nutrient composition of weight reduction diets. Food Chem 1996; 57:71-75.

74. Shimbo S, Zhang ZW, Miyate K, Watanabe T, Nakastsuka H, Matsuda-Inoguchi $\mathrm{N}$ et al. Estimates of mineral intakes using food composition tables vs measures by inductively-coupled plasma mass spectrometry: Part 1.Calcium, phosphorus and iron. Eur J Clin Nutr 1999; 53:226-232.
75. Shimbo S, Zhang ZW, Miyate K, Watanabe T, Nakastsuka H, Matsuda-Inoguchi $\mathrm{N}$ et al. Estimates of mineral intakes using food composition tables vs measures by inductively-coupled plasma mass spectrometry: Part 2.Sodium, potassium, magnesium, copper, zinc. Eur J Clin Nutr 1999; 53:233-238.

76. Rodríguez-Palmero M, Castellote-Bargalló AI, López-Sabater C, De la Torre-Boronat C, River-Urgell M. Assessment of dietary nutrient intakes: analysed vs calculated values. Food Chemistry 1998; 61:215-221.

77. Root AD, Toma RB, Frank GC, Reiboldt W. Meals identified as healthy choices on restaurant menus: an evaluation of accuracy. Int J Food Sci Nutr 2004;55:449-454.

78. Moreno-Torres R, Ruiz-López MD, Artacho R, Oliva P, Baena F, Baro L et al. Dietary intake of calcium, magnesium and phosphorus in an elderly population using duplicate diet sampling vs food composition tables. J Nutr Health Aging 2001; 5(4):253-255.

79. Contato R, Riso P, Ciapppellano S, Oriani GA, Testolin G. Nutritional evaluation of some processed catering foods. Int J Food Sci Nutr 2001; 52(1):7177.

80. Obarzanek E, Reed DB, Bigelow C, Glovsky E, Pobock R, Nicklas T et al Fat and sodium content of school lunch foods: Calculated values and chemical analysis. Int J Food Sci Nutr 1993; 44:155-165.

81. Sakurai K, Watanabe T, Matsuda-Inoguchi N, Shimbo S, Date C, Toji C et al. Agreement of estimated values with measured values in dietary intakes of minerals: A validation study in Japan. J Food Comp Anal 2008; 21:26-34.

82. Valsta L, Heinonen M, Anttoainen M, Mutanen M. Comparison of calculated and analyzed values: antioxidant and fatty acid composition. Food Chem 1996; 57:119-123.

83. Farran Codina A, Zamora Ros R. Tablas de composición de alimentos: aplicaciones en salud pública. En: Serra Majem LL, Aranceta Bartrina J. Nutri-

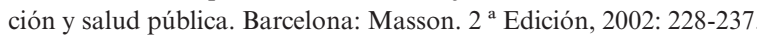

